\title{
The Shape of the Interstellar Reddening Curve ${ }^{1}$
}

\author{
ANNe B. Underhill and Gordon A. H. Walker \\ Dominion Astrophysical Observatory \\ Victoria, British Columbia, Canada
}

$\mathrm{W}$

HEN THE OBSERVED SPECTRAL INTENSITY DISTRIBUTION of an earlytype star has been freed from the distortions caused by absorption in the Earth's atmosphere and by instrumental sensitivity factors, it is a measure of the true spectral intensity distribution of the star and of the modifications to that distribution caused by the interstellar material between the Earth and the star. Thus, if one knew the true intensity distribution from the star, one could derive the shape of the interstellar reddening curve. In practice it is customary to compare the intensity distributions of pairs of stars of apparently the same spectral type, within the errors of classification, but which suffer different amounts of reddening, and to attribute the difference to interstellar reddening. In this way one can determine the shape of the interstellar reddening curve.

The results of reference 1 show that the monochromatic extinction, expressed in magnitudes, varies linearly with $\lambda^{-1}$ from ultraviolet wavelengths to about $4500 \AA$, where the slope of the curve appears to change and there is a second linear section to about $20000 \AA$. At still longer wavelengths, the extinction becomes independent of wavelength, more or less as suggested by theoretical studies of the extinction caused by small grains.

In recent years, model atmospheres in radiative equilibrium, which are believed to give a good representation of the parts of a stellar atmosphere in which the continuous spectrum is formed, have been obtained. This paper presents a comparison between the intensity distributions of some early $B$ and late $O$ type stars observed by the authors of references 2 and 3 and the intensity distributions from model atmospheres believed to represent $\mathrm{O} 9$ and $\mathrm{B} 0$ stars (refs. 4 and 5). Since the

\footnotetext{
${ }^{1}$ The contents of this paper were published previously in the Monthly Notices of the Roy. Astron. Soc., vol. 131, 1966, pp. 475-481.
} 
models are constructed using a well-defined procedure and their continuous spectrum can be calculated in any desired detail, the differences between the observed and computed intensity distributions can be established with precision.

By studying heavily reddened and lightly reddened stars, one can separate the effects due to interstellar reddening from those due to departures of the continuous spectrum of the stars from the predicted spectrum of the models. In particular, it should be possible to confirm or deny the existence of the "knee" in the reddening curve near $\lambda=4500$. This feature is difficult to account for with the present theories of the interaction between radiation and the interstellar grains. Some doubt exists about whether it is of interstellar origin or merely a reflection of differences of spectral type between the stars being compared. In addition, it would be reassuring to establish that the continuous spectrum of stars actually conforms to the predictions of the models. Since the predictions depend chiefly upon the variation of the adopted continuous absorption coefficients (H, He I, He II, and electron scattering) with wavelength, any serious departures from the predicted spectrum would necessitate a revision of the principles upon which the models have been constructed.

\section{OBSERVED INTENSITY DISTRIBUTIONS}

For the present purpose it is necessary only to study the relative intensity distribution in a stellar spectrum. The absolute intensity distributions given in reference 2 for $\zeta$ Ophiuchi, $09.5 \mathrm{~V}$, and $\epsilon$ Orionis, Bola, and those given in reference 3 for HD 154368 (O9.5Ia) and HD 167971 are used. (The spectral type listed for this star in reference 4 is O8f. However, spectrograms taken at the Dominion Astrophysical Observatory suggest a type near $\mathrm{B} 0 \mathrm{~V}$ in accord with classifications given in references 5 and 6.) Reference 3 gives data for $\zeta$ Ophiuchi which are in accord with those of reference 2. The observations of reference 2 extend from $3390 \AA$ to $10800 \AA$; those of reference 3 , from $4000 \AA$ to $6500 \AA$. The published data are converted into fluxes per angstrom relative to the flux at $5000 \AA$. Thus the intensity distribution in each star is represented by the function $F_{\lambda}(\lambda) / F_{\lambda}(5000 \AA)$.

\section{THEORETICAL INTENSITY DISTRIBUTIONS}

The observed relative intensity distributions will be compared with those predicted from models computed in references 7 and 8 . The computed continuous spectrum $F_{\nu}$ for five models is given in table I. The computations were performed using the Theoretical Spectrum Program. (See ref. 9.) Detailed data are given for the range from $2000 \AA$ to $12213 \AA$. The relative energy distributions $F_{\lambda}(\lambda) / F_{\lambda}(5000 \AA)$ are listed in table II. These data are the standards with which the observed relative intensity 
TABLE I. - Emergent Spectrum $\mathrm{F}_{v}$ of Five Models

\begin{tabular}{|c|c|c|c|c|c|}
\hline \multirow{2}{*}{$\lambda, \AA^{\circ}$} & \multicolumn{5}{|c|}{$F_{\nu}$, ergs $/ \mathrm{cm}^{2}-\sec -\left(\Delta_{\nu}^{\circ}\right)^{\mathrm{b}}$} \\
\hline & Model 63 & Model 89 & Model 97 & Model 64 & Model 98 \\
\hline 2000 & $2.5002 \times 10^{-3}$ & $3.3823 \times 10^{-3}$ & $4.5305 \times 10^{-3}$ & $5.1925 \times 10^{-3}$ & $6.7421 \times 10^{-3}$ \\
\hline 2200 & 2.2804 & 3.0781 & 4.1129 & 4.7434 & 6.2218 \\
\hline 2400 & 2.0869 & 2.7704 & 3.6991 & 4.2840 & 5.6005 \\
\hline 2600 & 1.9231 & 2.5166 & 3.3341 & 3.8556 & 5.0725 \\
\hline 2800 & 1.7869 & 2.3104 & 3.0351 & 3.4960 & 4.6123 \\
\hline 3000 & 1.6578 & 2.1299 & 2.7698 & 3.1784 & 4.1835 \\
\hline 3100 & 1.5950 & 2.0441 & 2.6517 & 3.0378 & 3.9941 \\
\hline 3200 & 1.5395 & 1.9646 & 2.5437 & 2.9091 & 3.8202 \\
\hline 3300 & 1.4849 & 1.8867 & 2.4331 & 2.7875 & 3.6509 \\
\hline 3400 & 1.4294 & 1.8145 & 2.3251 & 2.6731 & 3.4931 \\
\hline 3500 & 1.4044 & 1.7725 & 2.2615 & 2.5915 & 3.3663 \\
\hline 3600 & 1.3526 & 1.7013 & 2.1668 & 2.4868 & 3.2273 \\
\hline $3646 \mathrm{~V}$ & 1.3263 & 1.6658 & 2.1197 & 2.4348 & 3.1583 \\
\hline 3646R & 1.7716 & 2.1365 & 2.5775 & 2.8455 & 3.4428 \\
\hline 3700 & 1.7710 & 2.1256 & 2.5547 & 2.8121 & 3.3885 \\
\hline 3800 & 1.7049 & 2.0433 & 2.4538 & 2.7011 & 3.2539 \\
\hline 3900 & 1.6431 & 1.9639 & 2.3558 & 2.5961 & 3.1269 \\
\hline 4000 & 1.5821 & 1.8823 & 2.2586 & 2.4932 & 3.0074 \\
\hline 4200 & 1.4647 & 1.7360 & 2.0824 & 2.2975 & 2.7897 \\
\hline 4400 & 1.3600 & 1.6080 & 1.9270 & 2.1251 & 2.5894 \\
\hline 4600 & 1.2673 & 1.4949 & \begin{tabular}{|l|}
1.7892 \\
\end{tabular} & 1.9711 & 2.4037 \\
\hline 4800 & 1.1842 & 1.3939 & 1.6665 & 1.8339 & 2.2374 \\
\hline 5000 & 1.1104 & 1.3041 & 1.5557 & 1.7109 & 2.0861 \\
\hline 5200 & 1.0439 & 1.2234 & 1.4573 & 1.6011 & 1.9508 \\
\hline 5400 & .9830 & 1.1506 & 1.3674 & 1.5022 & 1.8297 \\
\hline 5600 & .9280 & 1.0859 & 1.2862 & 1.4125 & $\cdot 1.7203$ \\
\hline 5800 & .8776 & 1.0240 & 1.2128 & 1.3311 & 1.6226 \\
\hline 6000 & .8291 & .9653 & 1.1407 & 1.2567 & 1.5309 \\
\hline 6500 & .7251 & .8407 & .9874 & 1.0900 & 1.3321 \\
\hline 7000 & .6376 & .7364 & .8646 & .9564 & 1.1677 \\
\hline 7500 & .5653 & .6514 & .7633 & .8460 & 1.0301 \\
\hline 8000 & .5072 & .5823 & .6811 & .7542 & .9179 \\
\hline $8206 \mathrm{~V}$ & .4838 & .5553 & .6484 & .7195 & .8756 \\
\hline $8206 \mathrm{R}$ & .5150 & .5861 & .6767 & .7413 & .8926 \\
\hline 8700 & .4635 & .5263 & .6078 & .6672 & .8019 \\
\hline 9214 & .4164 & .4727 & .5453 & .5994 & .7208 \\
\hline 10503 & .3275 & .3700 & .4254 & .4695 & .5649 \\
\hline 12213 & .2460 & .2774 & .3192 & .3518 & .4231 \\
\hline
\end{tabular}

a $V$ denotes immediately toward the violet, and $R$ denotes immediately toward the red.

" $\nu^{\circ}$ is frequency in $(\AA)^{-1}$ 
TABLE II.-Relative Intensity Distributions $\mathrm{F}_{\lambda}(\lambda) / \mathrm{F}_{\lambda}(5000 \AA)$ of Five Models

\begin{tabular}{|c|c|c|c|c|c|c|}
\hline \multirow{2}{*}{$\lambda(\AA)$} & \multicolumn{5}{|c|}{$F_{\lambda}(\lambda) / F_{\lambda}(5000 \AA)$ for -} & \multirow{2}{*}{$\lambda^{-1}, \mu^{-1}$} \\
\hline & Model 63 & Model 89 & Model 97 & Model 64 & Model 98 & \\
\hline 2000 & 14.106 & 16.213 & 18.20 & 18.969 & 20.200 & 5.000 \\
\hline 2200 & 10.639 & 12.194 & 13.662 & 14.323 & 15.532 & 4.545 \\
\hline 2400 & 8.160 & 9.225 & 10.328 & 10.875 & 11.804 & 4.167 \\
\hline 2600 & 6.420 & 7.137 & 7.925 & 8.335 & 8.990 & 3.846 \\
\hline 2800 & 5.133 & 5.653 & 6.224 & 6.517 & 7.053 & 3.571 \\
\hline 3000 & 4.149 & 4.538 & 4.947 & 5.163 & 5.572 & 3.333 \\
\hline 3100 & 3.739 & 4.077 & 4.434 & 4.619 & 4.983 & 3.226 \\
\hline 3200 & 3.382 & 3.677 & 3.989 & 4.148 & 4.468 & 3.125 \\
\hline 3300 & 3.068 & 3.321 & 3.589 & 3.739 & 4.016 & 3.030 \\
\hline 3400 & 2.785 & 3.010 & 3.235 & 3.380 & 3.629 & 2.941 \\
\hline 3500 & 2.583 & 2.775 & 2.969 & 3.094 & 3.296 & 2.857 \\
\hline 3600 & 2.350 & 2.517 & 2.687 & 2.803 & 2.984 & 2.778 \\
\hline $3646 \mathrm{~V}$ & 2.247 & 2.402 & 2.562 & 2.675 & 2.845 & 2.742 \\
\hline $3646 \mathrm{R}$ & 3.000 & 3.080 & 3.114 & 3.166 & 3.103 & 2.742 \\
\hline 3700 & 2.911 & 2.975 & 2.997 & 3.000 & 2.964 & 2.703 \\
\hline 3800 & 2.659 & 2.714 & 2.731 & 2.735 & 2.705 & 2.632 \\
\hline 3900 & 2.433 & 2.476 & 2.489 & 2.494 & 2.464 & 2.564 \\
\hline 4000 & 2.226 & 2.254 & 2.268 & 2.276 & 2.252 & 2.500 \\
\hline 4200 & 1.868 & 1.885 & 1.896 & 1.902 & 1.893 & 2.381 \\
\hline 4400 & 1.580 & 1.591 & 1.598 & 1.602 & 1.601 & 2.273 \\
\hline 4600 & 1.349 & 1.355 & 1.359 & 1.362 & 1.362 & 2.174 \\
\hline 4800 & 1.158 & 1.161 & 1.163 & 1.164 & 1.165 & 2.083 \\
\hline 5000 & 1.000 & 1.000 & 1.000 & 1.000 & 1.000 & 2.000 \\
\hline 5200 & .869 & .867 & .866 & .865 & .864 & 1.923 \\
\hline 5400 & .759 & .757 & .754 & .753 & .752 & 1.852 \\
\hline 5600 & .666 & .664 & .659 & .658 & .657 & 1.786 \\
\hline 5800 & .587 & .584 & .579 & .578 & .578 & 1.724 \\
\hline 6000 & .519 & .514 & .509 & .510 & .510 & 1.667 \\
\hline 6500 & .386 & .382 & .376 & .377 & .378 & 1.538 \\
\hline 7000 & .293 & .288 & .284 & .285 & .286 & 1.429 \\
\hline 7500 & .226 & .222 & .218 & .220 & .220 & 1.333 \\
\hline 8000 & .178 & .174 & .171 & .172 & .172 & 1.250 \\
\hline $8206 \mathrm{~V}$ & .162 & .158 & .155 & .156 & .156 & 1.219 \\
\hline 8206R & .172 & .167 & .162 & .161 & .159 & 1.219 \\
\hline 8700 & .138 & .133 & .129 & .129 & .127 & 1.149 \\
\hline 9214 & .110 & .107 & .103 & .103 & .102 & 1.085 \\
\hline 10503 & .067 & .064 & .062 & .062 & .061 & .952 \\
\hline 12213 & .037 & .036 & .034 & .034 & .034 & .819 \\
\hline
\end{tabular}


distributions are compared. The models are all considered to represent main-sequence stars. Their equivalent spectral types are:

\begin{tabular}{ccc} 
Model & \multicolumn{3}{c}{ Spectral type } \\
63 & $\ldots \ldots \ldots \ldots \ldots \ldots \ldots$ & \multicolumn{1}{c}{ B2 } \\
89 & $\ldots \ldots \ldots \ldots \ldots \ldots \ldots$ & B1 \\
97 & $\ldots \ldots \ldots \ldots \ldots \ldots \ldots$ & B0.5 \\
64 & $\ldots \ldots \ldots \ldots \ldots \ldots \ldots$ & B0 \\
98 & $\ldots \ldots \ldots \ldots \ldots \ldots \ldots$ & O9
\end{tabular}

\section{COMPARISON BETWEEN THEORY AND OBSERVATION}

The differences between the observed distributions and that for model 98 at each selected wavelength are given in tables III and IV. A negative magnitude difference means that the star is brighter than the model by the given amount, the fluxes in each object, star, or model being measured in units of the flux at $5000 \AA$. A positive magnitude difference means that the star is fainter than the model at the listed wavelength.

TABLE III.-Magnitude Differences Between Model 98 and Observed Intensity Distributions of $\zeta$ Ophiuchi and $\epsilon$ Orionis

\begin{tabular}{|c|c|c|c|c|c|c|c|}
\hline \multirow[t]{2}{*}{$\lambda, \AA$} & \multirow[t]{2}{*}{$\lambda^{-1}, \mu^{-1}$} & \multicolumn{2}{|c|}{$\begin{array}{l}\text { Magnitude difference } \\
\text { between model } 98 \\
\text { and- }\end{array}$} & \multirow[t]{2}{*}{$\lambda, \AA$} & \multirow[t]{2}{*}{$\lambda^{-1}, \mu^{-1}$} & \multicolumn{2}{|c|}{$\begin{array}{c}\text { Magnitude difference } \\
\text { between model } 98 \\
\text { and - }\end{array}$} \\
\hline & & $\zeta O \mathrm{ph}$ & $\epsilon$ Ori & & & $\zeta$ Oph & $\epsilon$ Ori \\
\hline 3390 & 2.950 & +0.455 & +0.160 & 5840 & 1.712 & -0.225 & -0.115 \\
\hline 3448 & 2.900 & +0.425 & +0.132 & 6055 & 1.652 & -0.272 & -0.142 \\
\hline 3509 & 2.850 & +0.402 & +0.132 & 6370 & 1.570 & -0.325 & -0.152 \\
\hline 3571 & 2.800 & +0.395 & +0.122 & 6800 & 1.471 & -0.418 & -0.218 \\
\hline 3636 & 2.750 & +0.350 & +0.098 & 7100 & 1.408 & -0.498 & -0.255 \\
\hline 3704 & 2.700 & +0.498 & +0.232 & 7530 & 1.328 & -0.550 & -0.292 \\
\hline 4032 & 2.480 & +0.310 & +0.152 & 7850 & 1.274 & -0.603 & -0.303 \\
\hline 4167 & 2.400 & +0.268 & +0.132 & 8080 & 1.238 & -0.638 & -0.338 \\
\hline 4255 & 2.350 & +0.242 & +0.102 & 8400 & 1.190 & -0.670 & -0.350 \\
\hline 4464 & 2.240 & +0.198 & +0.092 & 8805 & 1.136 & -0.675 & -0.335 \\
\hline 4566 & 2.190 & +0.152 & +0.062 & 9700 & 1.031 & -0.758 & -0.353 \\
\hline 4787 & 2.090 & +0.072 & +0.032 & 9950 & 1.005 & -0.790 & -0.410 \\
\hline 5000 & 2.000 & +0.000 & +0.000 & 10250 & .976 & -0.838 & -0.448 \\
\hline 5263 & 1.900 & -0.088 & -0.038 & 10400 & .962 & -0.835 & -0.435 \\
\hline 5556 & 1.800 & -0.165 & -0.080 & 10800 & .926 & -0.868 & -0.468 \\
\hline
\end{tabular}

The relative intensity distributions of models 98 and 64 are indistinguishable between $4000 \AA$ and $6500 \AA$; thus it suffices to compare the observations with one model. The spectrum from model 98 is significantly brighter than that from model 64 at wavelength shorter than the Balmer limit. 
TABle IV.-Magnitude Differences Between Model 98 and Observed Intensity Distributions of HD 154368 and HD 167971

\begin{tabular}{|c|c|c|c|}
\hline \multirow{2}{*}{$\lambda, \AA$} & \multirow{2}{*}{$\lambda^{-1}, \mu^{-1}$} & \multicolumn{2}{|c|}{$\begin{array}{l}\text { Magnitude difference between } \\
\text { model } 98 \text { and - }\end{array}$} \\
\hline & & HD 154368 & HD 167971 \\
\hline 4000 & 2.500 & +0.798 & +1.028 \\
\hline 4200 & 2.381 & +0.670 & +0.839 \\
\hline 4400 & 2.273 & +0.505 & +0.639 \\
\hline 4600 & 2.174 & +0.344 & +0.396 \\
\hline 4800 & 2.083 & +0.172 & +0.236 \\
\hline 5000 & 2.000 & +0.000 & +0.000 \\
\hline 5200 & 1.923 & -0.164 & -0.204 \\
\hline 5400 & 1.852 & -0.299 & -0.385 \\
\hline 5600 & 1.786 & -0.436 & -0.571 \\
\hline 5800 & 1.724 & -0.509 & -0.681 \\
\hline 6000 & 1.667 & -0.654 & -0.849 \\
\hline 6500 & 1.538 & -0.909 & -1.177 \\
\hline
\end{tabular}

From each set of data the color excess between the $V$ and $B$ points $\left(\lambda^{-1}=1.83\right.$ and $\lambda^{-1}=2.29$, respectively) was found. It is 0.361 magnitude for $\zeta$ Ophiuchi, 0.180 magnitude for $\epsilon$ Orionis, 0.870 magnitude for HD 154368, and 1.110 magnitudes for HD 167971. The data were then normalized to an excess of 1.000 magnitude between $V$ and $B$, and the points were plotted to give the composite reddening curve shown in figure 1 .

Four conclusions are immediately evident:

1. The comparison with theoretical spectra has produced a reddening curve like that found by standard observational procedures. Consequently, it may be concluded that over the range from $4000 \AA$ to $6500 \AA$ at least, the spectrum from model 98 represents well the spectra from real stars of a type near 09 .

2. A definite "knee" occurs in the reddening curve. The break appears to occur at $\lambda^{-1}=2.25$ (4450 $\AA$ ).

3. If the decrease in gradient for $\lambda^{-1}>2.25$ is due to an anomalous brightening of the stellar spectrum and not to weaker interstellar extinction, the gradient for $\lambda^{-1}>2.25$ should be a function of color excess. This is not true, which means that the "knee" is not due to an anomaly in the stellar spectrum.

4. The stars $\zeta$ Ophiuchi and $\epsilon$ Orionis are considerably brighter in the Balmer continuum and in the Paschen continuum than predicted by the model. They appear to be surrounded by extended envelopes 


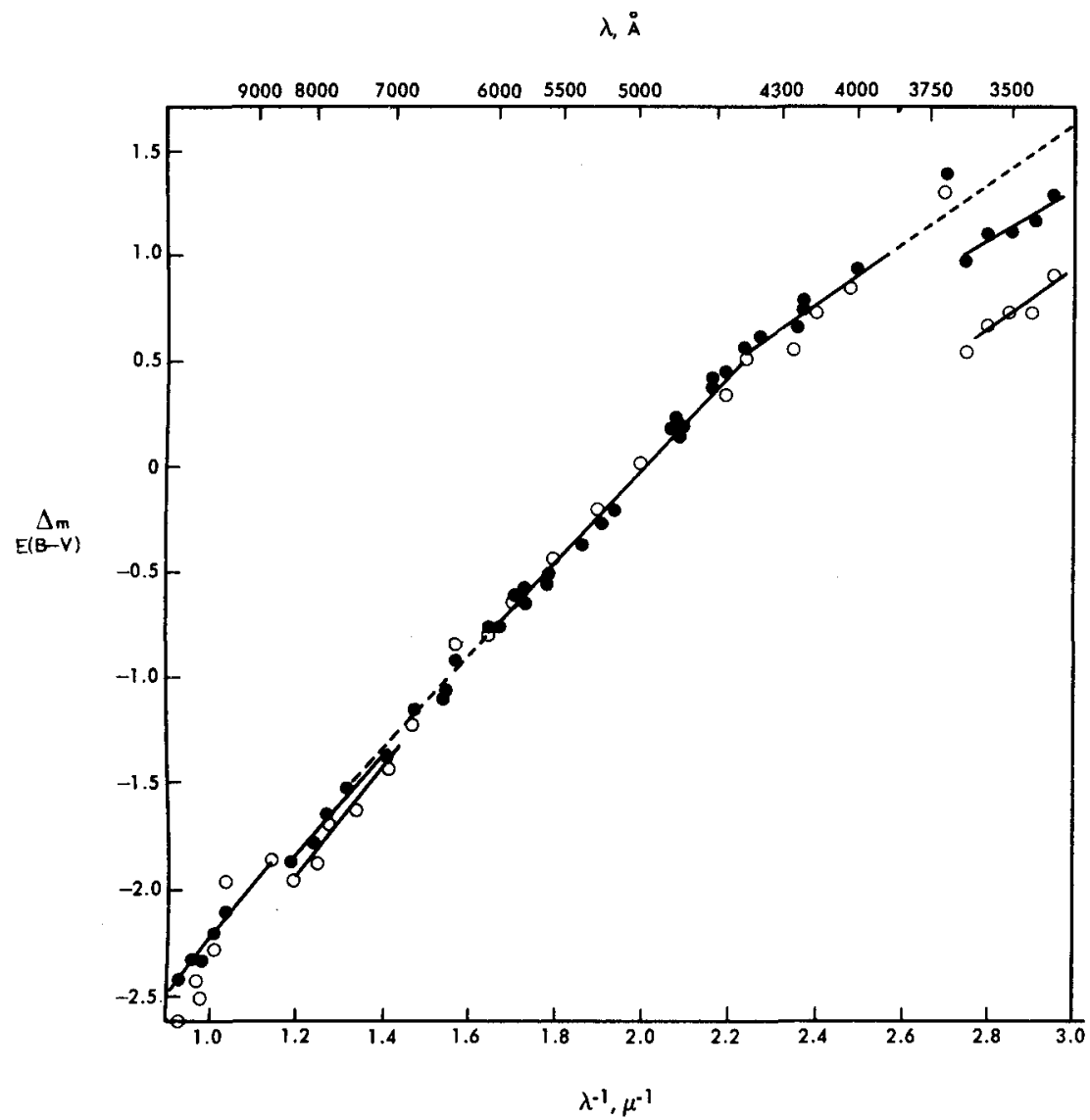

FIGURE 1.-Normalized interstellar reddening curve. Closed circles, HD 154368 and HD 167971 for $4000 \AA<\lambda<6500 \AA$, and $\zeta$ Ophiuchi, for all values of $\lambda$; open circles, $\epsilon$ Orionis.

which give the Balmer continuum in emission, a not unlikely state of affairs for such early-type stars.

In the discussion the point at $\lambda^{-1}=2.700(3704 \AA)$ is ignored because the overlapping of the Balmer lines probably depresses the stellar continuum there.

The normalized reddening lines may be represented by the following equations:

$$
\begin{aligned}
& \Delta m_{\lambda}=2.23(1 / \lambda-4.46) \text { for } 2.25>\lambda^{-1}>1.45 \\
& \Delta m_{\lambda}=1.42(1 / \lambda-2.65) \text { for } 2.25<\lambda^{-1}<2.50
\end{aligned}
$$

These results may be compared with those of reference 10 . The equations in this reference are normalized to give unit gradient for $2.25<\lambda^{-1}$, and $\Delta m_{\lambda}=1$ for $\lambda^{-1}=2.22$. In this reference is found

$$
\Delta m_{\lambda}=\lambda^{-1}-1.22 \quad \text { for } 1.0 \leqslant \lambda^{-1}<2.30
$$




$$
\Delta m_{\lambda}=0.64\left(\lambda^{-1}-0.39\right) \text { for } \lambda^{-1}>2.30
$$

Applying the same normalization conditions to equations (1) and (2) gives:

$$
\begin{aligned}
& \Delta m_{\lambda}=\lambda^{-1}-1.22 \quad \text { for } 2.25>\lambda^{-1}>1.45 \\
& \Delta m_{\lambda}=0.64\left(\lambda^{-1}-0.41\right) \text { for } 2.25<\lambda^{-1}<2.50
\end{aligned}
$$

The agreement is excellent and strongly supports conclusions 1 and 3 given previously. Furthermore since the four stars being studied do not lie in the Cygnus region, but are scattered around the sky, the agreement between the reddening law found here and that of reference 10 lends support to the idea that the scattering properties of interstellar grains are the same in all parts of the galaxy.

\section{REFERENCES}

1. Sharpless, S.: Interstellar Reddening. Stars and Stellar Systems, vol. 3, Strand, ed., University of Chicago Press, 1963, p. 225.

2. OKE, J. B.: Photoelectric Spectrophotometry of Stars Suitable for Standards. Astrophys. J., vol. 140, 1964, p. 689.

3. Willstrop, R. V.: Absolute Measures of Stellar Radiation II. Roy. Astron. Soc., Memoirs, vol. 69, pt. 3, 1965.

4. Morgan, W. W.; Code, A. D.; and Whitrord, A. E.: Studies in Galactic Structure II. Luminosity Classification for 1270 Blue Giant Stars. Astrophys. J., Suppl. Ser., vol. 2, 1955, p. 41.

5. Neubauer, F. J.: The Radial Velocities of Faint Class B Stars in the Declination Zone $0^{\circ}$ to $-23^{\circ}$. Astrophys. J., vol. 97, 1943, p. 300.

6. SANFoRD, R. F.: Interstellar Calcium Lines in the Spectra of Stars in Open Clusters. Astrophys. J., vol. 110, 1949, p. 117.

7. Underhill, A. B.: A Program for Computing Early-Type Model Atmospheres and Testing the Flux Integral. Pub. Dominion Astrophys. Obs., vol. II, no. 23, 1962.

8. UNDERHILl, A. B.: Concerning the Interpretation of Line Strengths in B-Type Spectra. Bull. Astron. Inst. Netherlands, vol. 17, 1963, p. 161.

9. Underhill. A. B.: A Program for Computing the Theoretical Spectrum from a Model Atmosphere. Pub. Dominion Astrophys. Obs., vol. II, no. 24, 1962.

10. Nandy, K.: Observations of Interstellar Reddening I. Results for Region in Cygnus. Edinburgh Pub., vol. 3, no. 6, 1964.

\section{DISCUSSION}

Wickramasinghe: I would like to point out that the knee in Dr. Nandy's curve occurs rather strikingly at a wavelength where the refractive index for graphite begins to change considerably; from the infrared up to that point it remains reasonably constant, and then begins to vary.

Greenberg: Is there any good reason why the graphite index should change sufficiently to make such a drastic change in the extinction? These are two straight lines. 
Wickramasinghe: What $I$ have done is to perform Mie computations for graphite by using recently available refractive index data. For radii less than about $0.06 \mu$ the extinction efficiency curves have the property of remaining closely like the curves for $\lambda^{-1}$ in the wavelength range $0.8<\lambda^{-1}<2.4$, and thereafter deviating considerably from the $\lambda^{-1}$ curve. The precise structure of the curves for shorter wavelengths appears to depend sensitively on the particle size. If $I$ take a size distribution of graphite, I can simulate approximately Dr. Nandy's twostraight-line effect.

Greenberg: Then this effect will show up if there are many small graphite particles corresponding to this type of extinction?

Wickramasinghe: Yes. The straight-line segment for $\lambda^{-1}<2.4$ could be obtained almost independently of size-provided it is less than about $0.06 \mu$. But, in order to get a specified ratio of slopes over the two segments, one has to be a bit careful in mixing sizes.

Greenberg: Why can this be observed in Perseus as well as in Cygnus? In one case one has pure graphite. Then I would expect that if something happened to the graphite that is pure and small, this effect would be observed.

If on the other hand one had to account for this large extinction in the UV by putting on dirty ice mantles, which we have had to do, these ice mantles or whatever we put on would then be sufficiently large to obscure this slope discontinuity. However, if I remember correctly, Dr. Nandy's curves had a change in slope with a rather sharp discontinuity, both for Perseus as well as for Cygnus. Can you explain these two?

Wickramasinghe: Yes; the point is that if we take the graphite core radius of the order of 2 to $3 \times 10^{-6} \mathrm{~cm}$ and cover this core with an ice mantle of up to 1.5 times that radius, then 60 percent of the extinction in the visible and the ultraviolet comes from the graphite core. The reason is that one has to have a value of $(2 \pi a) / \lambda$ of the order of unity where $a$ is the outer mantle radius before one can get a significant contribution from the ice mantle. For example, if you have an ice grain with a radius of $0.05 \mu$, you won't get very much absorption in the visible compared with what you would obtain with a graphite core of radius $0.03 \mu$.

Greenberg: I disagree. We have computed also for $0.05-\mu$ graphite cores covered with ice mantles of small as well as large dimensions and do not find this to be true.

Wickramasinghe: It depends on how much ice mantle you have.

Greenberg: I assume that the ice mantles are sufficient to give one good extinction in the UV. I think, however, that this is a very fundamental point, at least as fundamental as the character of the extinction at $2400 \AA$. 
Wickramasinghe: At least it is the case in comparing the extinction cross section for an ice particle of radius $0.05 \mu$ with that for a graphite core of radius $0.03 \mu$ that the ice absorption is very much smaller. If one compares the cross section for the composite particle with that for the core itself, it turns out that the core absorption amounts to about 80 percent in the infrared, 70 percent in the visible, and 60 percent in the blue.

Borgman: Miss Underhill has criticized sometimes the practice of comparing stars of equal spectral classification with the purpose of deriving their reddening properties. Now I note with some satisfaction that you find the same results as these which were critical. Does this now satisfy you and Miss Underhill that the previous practices that have been used are satisfactory?

Walker: I don't quite understand. Did she normally criticize the use of stars which do not necessarily have exactly the same spectrum?

Borgman: Yes, and I do not think that the stars which you have analyzed here have the same spectral type.

Walker: No, I quite agree. I didn't get the impression when I was writing this with Miss Underhill that she felt there should be a change in viewpoint.

Borgman: The fact that you find the same results is at least encouraging.

Walker: Yes, I think one has to make a proviso that we have stipulated the range over which this has been satisfactory. There is no question that we can use this where continua are concerned. I think that obviously we must be quite cautious about anything which might involve shell emission. As I said before, this is the hottest stellar model Miss Underhill has computed so far and it looks as if it is satisfactory in the visual, ultraviolet, and red. I think we are bringing up the point of graphite too early. It might be worth deciding just how sharp the discontinuity of slope is. As far as I could make out in our work it was just a complete break. Now, we obviously do not have sufficient resolution to state that categorically. I don't know how you would find it in your results.

Nandy: It is difficult to say how sharp this discontinuity is from my results because the dispersion is too low. Another program is in progress using 60 - and $120-\AA$ dispersions, and the same stars will be studied; we hope to determine how sharp this change in slope is.

Greenberg: I wonder if Dr. Walker has any comments. This happens to be around 4430 .

Walker: In the way that I drew it I found that it could coincide with the wavelength of 4430 . But $I$ don't think it is going to have anything to do with 4430. I think that one would expect for unit color excess a central depth of the order of 10 percent for the 4430 line. With the $50 . \AA$ resolu- 
tion used in scanner results, one would not see the full 10 percent, but would probably see about 1 percent. This becomes something like onehundredth of a magnitude and the discrepancy here, if one wants to draw in a smooth curve, is more of the order of one-tenth of magnitude.

Wampler: I would agree, I think. The wavelength 4430 is not the. agent here.

Olson: One of your stars is very obviously a rapid rotator $-\zeta$ Ophiuchi; I think it is about $400 \mathrm{~km} / \mathrm{sec}$.

Walker: The apparent Balmer and Paschen emission might be due to that.

Wickramasinghe: Could you say something about how these stars are distributed in the galaxy?

Walker: There is one star in Orion and there are three in the Southern Hemisphere reasonably close together. I think Dr. Nandy showed them on his graph. Actually, I think it is a pity that we didn't proceed and go through the whole of Willstrop's list (ref. 3). But, of course, we couldn't do it in this way. I think probably one could go about it now in what I would call the classical fashion.

Field: Could I ask about the bump in the extinction at $\lambda=6300$ ? Do you believe that?

Walker: I don't really know; it just happens to occur in both our graphs. It happens to be a kink in the same direction as far as our experimental errors are concerned. It looks like a significant thing. There is no strong interstellar absorption band there, and $\lambda=6284$ is some distance away, but again this is something $I$ just noticed in passing.

Wampler: We were doing some work at Lick in regard to stellar energy distributions, and we have had to find out what the extinction of the atmosphere is. It follows the curve for $\lambda^{-4}$ very closely to about $5500 \AA$ to $5800 \AA$; beyond that we quickly run into bumps and I wonder if there are regions in the atmosphere that might cause extinction in this spectral region. The extinction may not go as the secant of the altitude angle $z$ region.

Walker: But surely there should be a scatter at this point. It should not be all in the same direction.

Wampler: All your stars with the exception of Orion are in the Southern Hemisphere, which means that they never pass overhead.

Walker: They were all measured at the same zenith distance, but Dr. Nandy's results show that there should only be a scatter around that point. His standard stars and his reddened stars presumably do not have a systematic difference in $\sec z$.

Nandy: Yes, when they are on the same objective prism plates atmospheric extinction is not that important. But, when the reddened and comparison stars are not on the same plate, magnitude differences have beèn corrected for atmospheric extinction. 